Revue belge de géographie

\title{
Entre régionalisme et régionalisation, le difficile positionnement du Norden en Europe
}

Jacques Guillaume

\section{Q OpenEdition}

1 Journals

Édition électronique

URL : http://journals.openedition.org/belgeo/43803

DOI : $10.4000 /$ belgeo.43803

ISSN : 2294-9135

Éditeur :

National Committee of Geography of Belgium, Société Royale Belge de Géographie

Référence électronique

Jacques Guillaume, «Entre régionalisme et régionalisation, le difficile positionnement du Norden en Europe », Belgeo [En ligne], 4 | 2020, mis en ligne le 09 novembre 2020, consulté le 11 novembre 2020. URL : http://journals.openedition.org/belgeo/43803; DOI : https://doi.org/10.4000/belgeo.43803

Ce document a été généré automatiquement le 11 novembre 2020.

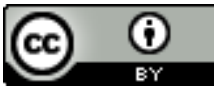

Belgeo est mis à disposition selon les termes de la licence Creative Commons Attribution 4.0 International. 


\title{
Entre régionalisme et régionalisation, le difficile positionnement du Norden en Europe
}

\author{
Jacques Guillaume
}

En première approche, il convient de préciser ce qu'on entend dans cet article par " régionalisme » et " régionalisation », tant ces termes sont mal stabilisés et font l'objet de glissements de sens permanents (Mareï, Richard, 2018). Nous estimons que le régionalisme doit désigner un état ou un processus conscient et volontaire de vivre ensemble, donc d'inspiration politique, mais dont les fondements peuvent être de nature diverse, culturelle ou économique. Il s'inscrit dans l'espace en décalage par rapport au pavage des États et peut donc à ce titre entrer en conflit avec lui, cette question se réglant par des solutions politiques (par décentralisation du pouvoir à l'échelle infraétatique, par coopération ou transfert de souveraineté à l'échelle supraétatique). La régionalisation en revanche signifie un processus de différenciation spatiale dont les principes sont ceux de l'homogénéisation (par l'extension de caractères communs) ou ceux de l'intégration (par la polarisation). Les processus à l'œuvre sont donc essentiellement économiques et sont plus faciles à appréhender par leurs effets que par leurs causes, souvent souterraines, spontanées et progressives. Les régions qui en résultent sont inclusives de leurs marges, en proportion de l'intensité des processus qui les ont fait naître. Elles peuvent être multiscalaires, se chevaucher les unes les autres, sans déboucher forcément sur un régionalisme d'ordre politique. Mais elles peuvent contrarier ou à l'inverse renforcer l'ordonnancement impulsé par le régionalisme; tout dépend bien sûr de l'état de concordance socio-spatiale de ces régions avec les structures régionalistes précédemment évoquées. Or, il apparaît que le Norden, c'est-à-dire l'ensemble regroupant les quatre États scandinaves (Danemark, Islande, Norvège et Suède) auquel s'ajoute la Finlande, est un cas d'école pour étudier le tiraillement possible entre régionalisme et régionalisation. Si le régionalisme se nourrit d'un ensemble qui paraît aller de soi pour des raisons géographiques, historiques et 
culturelles, il n'a rien donné de très solide, malgré les espoirs des plus ardents défenseurs du scandinavisme au XIX ${ }^{\text {ème }}$ siècle, puis du Conseil nordique au XX ${ }^{\text {ème }}$ siècle. C'est que d'autres principes d'organisation spatiale sont à l'œuvre, à ranger dans les catégories de la régionalisation. Ces principes peuvent se comprendre par l'intégration à des foyers externes de polarisation, derrière lesquels apparaît de manière sousjacente l'appartenance à l'Union européenne, effective pour seulement trois des cinq États en cause. Il convient ici de bien faire la distinction entre le continent européen dont le Norden fait incontestablement partie, surtout si on écarte les dépendances d'«outre-mer » du Danemark (Féroé et surtout Groenland) et l'Union européenne à laquelle le Norden met régulièrement en débat son appartenance, si l'on en juge par les consultations référendaires qui se sont déroulées de 1972 à 2015 . Ce sont d'ailleurs les résultats de ces consultations qui ont été la matière première initiale de notre propos, consolidé par la suite par la convocation d'un contexte historique de plus grande amplitude, tout en suggérant quelques interprétations d'ordre géographique qui mériteraient bien sûr d'être validées par un travail d'enquêtes à réaliser sur le terrain. En tout état de cause, notre postulat serait donc de rendre compte des limites du régionalisme nordique par des formes discordantes de régionalisation, ces formes de régionalisation se heurtant en revanche aux résistances nordiques pour empêcher une pleine adhésion au projet politique européen. Ce postulat, pour être soutenu et surtout compris, s'inscrit dans les schémas du «constructivisme " régional, dont quelques auteurs ont tenté de cerner les étapes (Hettne, Söderbaum, 2000), ces étapes pouvant être contrariées par des champs de forces centrifuges s'opposant à la construction d'une région distincte de son environnement extérieur.

\section{Un régionalisme ne débouchant sur aucune intégration politique définitive}

\section{Le rêve déçu du scandinavisme}

2 R. Boyer, dans un ouvrage collectif paru en 1992, évoquait le scandinavisme en ces termes: "le thème de l'unité scandinave est une des constantes immémoriales de l'histoire $\mathrm{du}$ Nord, ses premières manifestations remontant bien avant l'éphémère union de Kalmar, dès le Haut Moyen Âge en fait. L'âge romantique va fournir, au hasard de ses élans idéologiques ou proprement historiques, d'excellentes occasions d'incarner dans les faits ce vieux rêve - puisqu'il faut l'appeler ainsi. Or, nous constatons que rêve il fut, rêve il demeurera, alors que l'Histoire aura tout fait, si l'on peut dire, pour qu'il devienne réalité ». On ne peut mieux résumer à la fois l'élan et l'échec du scandinavisme dont les prétentions au XIX ${ }^{\text {ème }}$ siècle devaient tout naturellement conduire à la construction d'un État fédératif nordique, transcendant les divers microcosmes nationaux (Zorgbibe, 1968).

Ce mouvement prend naissance dans les années 1840, porté par des intellectuels, universitaires et étudiants, et relayé par les chancelleries et singulièrement, l'union des couronnes norvégienne et suédoise à partir de 1814 (Fol, 1978). Il débouche sur quelques mesures concrètes, dont les plus remarquables restent la fondation de l'association nationale nordique en 1865 et surtout de l'union monétaire nordique en 1873 (rejointe par la Norvège en 1875) dont les principes furent d'harmoniser les noms des diverses monnaies nationales, ainsi que d'en définir pour chacune une valeur or. 
Néanmoins, ces œuvres consistaient déjà à soutenir une idée presque moribonde, puisque le projet d'union politique avait sombré dès la fin des années 1860, avec l'affaire des Duchés, dans laquelle le Danemark s'était retrouvé seul face à la Prusse, malgré les promesses de soutien de la Suède. Aussi, le Danemark, cruellement amoindri sur le plan territorial, put mesurer à l'époque le profond égoïsme des pays « frères » et faire le deuil des grandes idées du fédéralisme scandinave. Beaucoup d'intellectuels purent alors tempêter contre la lâcheté et le verbiage de leurs compatriotes, comme $\mathrm{H}$. Ibsen dans un poème connu sous le titre "Un frère dans la détresse ", le mal était fait et la Scandinavie fut ainsi empêchée de suivre le destin de l'Allemagne ou de l'Italie, nées pourtant toutes les deux de multiples particularismes locaux et régionaux à peu près au même moment.

\section{La résurgence imparfaite d'un projet nordique}

4 La résurgence d'un projet nordique après la Seconde Guerre mondiale tient donc presque du miracle quand on sait le sort divergent des différents pays en cause au cours des années troubles des deux conflits mondiaux. C'est surtout la Finlande, libérée de la tutelle russe en 1917, mais à nouveau entravée par les contraintes imposées par l'Union soviétique, suite à la signature du traité de Paris de 1947 et de son traité d'amitié avec l'URSS en 1948, qui eut le chemin le plus difficile et le plus tortueux pour raccrocher les wagons d'une union nordique en 1955-1956, les pays scandinaves se regroupant derrière le drapeau du Conseil nordique, créé dès 1952, et baptisé comme tel dans la perspective précisément d'intégrer la Finlande, pays incontestablement nordique mais si peu scandinave, à la seule exception peut-être de sa population suédophone, appelée à se marginaliser progressivement dans le giron finlandais.

Ce Conseil, né d'une union interparlementaire qui avait été créée dès 1906, a beaucoup œuvré pour la cause nordique (Simoulin, 2003). Ses prérogatives ont été précisées par le traité d'Helsinki en 1962, en matière juridique, économique, sociale et culturelle. Ce traité a été refondu en 1971, faisant naître à l'occasion un conseil des ministres nordique, ses compétences étant élargies au fur et à mesure de la signature de conventions ultérieures, de sorte qu'il existe aujourd'hui onze conseils spécialisés, aux côtés de l'assemblée du conseil, regroupant 87 délégués des divers pays nordiques, ainsi que des territoires plus ou moins autonomes du Groenland, des Féroé et des îles Åland. Il est difficile dans le cadre de cet article de dresser un bilan détaillé du Conseil nordique, sauf pour dire qu'il s'est progressivement substitué à une coopération spontanée, en multipliant des organes de coopération, en particulier dans le domaine culturel. Ses acquis les plus spectaculaires restent cependant l'œuvre entreprise dès les années 1950 en matière d'ouverture des frontières pour la circulation et le droit d'installation et de travail de tous les citoyens nordiques dans les divers territoires des États concernés, sans parler des efforts d'harmonisation en matière de transports, de communication, de protection de l'environnement. Grâce au Conseil, des politiques régionales à propos de régions transnationales sont devenues réellement possibles, comme pour l'Øresund, région métropolitaine de Copenhague-Malmö sur les deux rives du détroit du même nom (Cabouret, 1992 ; Nauwelaers et al., 2013), ou pour la Calotte nordique, dans les périphéries septentrionales de la Norvège, de la Suède et de la Finlande (Cabouret, 1989). 


\section{Les ciments du régionalisme}

6 De telles avancées du régionalisme politique ne peuvent naturellement résulter que de facteurs convergents, au niveau des États comme à celui des sociétés nordiques. Il est notoire en effet de constater que ces sociétés se rejoignent par des patrimoines qui leur sont communs. Déjà, l'isolement et le confinement géographiques face à une nature difficile impriment leurs effets sur toutes ces populations, à la seule exception sans doute de la population danoise. Du reste, les Nordiques se rendant à Copenhague disent bien qu'ils vont sur le continent, tant ils ont l'impression de vivre dans un monde insulaire, alors même que la capitale danoise est précisément sur une île mais très densément peuplée, tandis que les Nordiques sont des continentaux, mais à l'écart de tout! Cette inversion des perceptions par rapport aux réalités géographiques les plus élémentaires, traduit bien l'idée d'eux-mêmes qu'ont les Nordiques. Ajoutons pour toutes ces populations, à la seule exception des Sames, de multiples caractères socioculturels qui les façonnent dans un moule identique : le culte de l'ancêtre viking et de ses vertus plus ou moins revisitées par l'Homme moderne, le sillon profond laissé par la Réforme, le cousinage linguistique facilitant l'intercompréhension des peuples et de leur culture, à la seule exception des Finlandais non suédophones, la nostalgie de la vie simple du monde rural, pour ces populations tardivement aspirées par la ville et qui retournent à la "hytte » chaque week-end, le sentiment enfin d'avoir réussi le " välfärdstat ", l'État-providence, et de pouvoir jouir de ses bienfaits, à un point tel que l'étranger l'admire sous le titre enviable de "modèle suédois" (Parent, 1970). Ce modèle, qu'il vaudrait mieux appeler "miracle suédois", tant il est spécifique au monde nordique, est le résultat d'interférences systémiques entre les trois pôles syndical, patronal et politique, dont les fondements remontent aux accords de Saltsjöbaden de 1938 entre le patronat suédois et la centrale syndicale LO. Sans en détailler les différentes orientations, on peut affirmer qu'il s'agit d'une forme originale de compromis entre le capital (dont personne ne revendique l'appropriation collective) et le travail, à propos d'objectifs convergents entre employeurs et salariés: une croissance économique forte, soutenue par des secteurs ouverts sur le monde et compétitifs, grâce à l'abondance d'intrants matériels nationaux, une politique de salaires élevés encourageant la recherche d'une forte productivité, une solidarité entre secteurs ouverts et secteurs " abrités » mise en musique par le parti social-démocrate, au pouvoir dans la longue durée. Ainsi, ce parti est aux affaires en Suède de 1932 à 1976, avec des personnalités d'une exceptionnelle longévité politique (Tage Erlander a été Premier ministre en Suède de 1946 à 1969). Les autres pays scandinaves ont suivi les mêmes stratégies et adopté des comportements politiques équivalents, donnant ainsi l'impression de l'unité du «bloc scandinave ». Les résultats de ces stratégies originales sont indéniables, tant sur le plan économique que sociétal. Pour ne prendre que le seul IDH, l'un des indicateurs le plus révélateur des niveaux de développement d'une société, la Norvège est au premier rang mondial en 2013, le Danemark au dixième rang et la Suède au douzième (la France est au $20^{\text {ème }}$ rang et la Belgique au $21^{\text {ème)}}$. Ce bloc de haut niveau de vie est donc fier de ses résultats, acquis de manière endogène. Précisons enfin qu'aucune guerre n'est venue alimenter l'inimitié entre les peuples nordiques, depuis l'été 1814 et le conflit éphémère entre Norvégiens et Suédois, conclu par la convention de Moss, soldant ainsi les griefs nés de l'union imposée, dès le début de la même année, entre les couronnes suédoise et norvégienne par le traité de Kiel. Rappelons enfin que les constructions territoriales, observées dans la longue durée 
depuis le Moyen Âge, ont grandement facilité l'entremêlement des peuples (tableau 1), même s'il fut parfois la cause de quelques rancœurs de la part des nations les plus tardivement venues à l'indépendance au XX ${ }^{\text {ème }}$ siècle (Norvège, Finlande et Islande).

Tableau 1. Les ensembles territoriaux de la fin du XIVème siècle à nos jours.

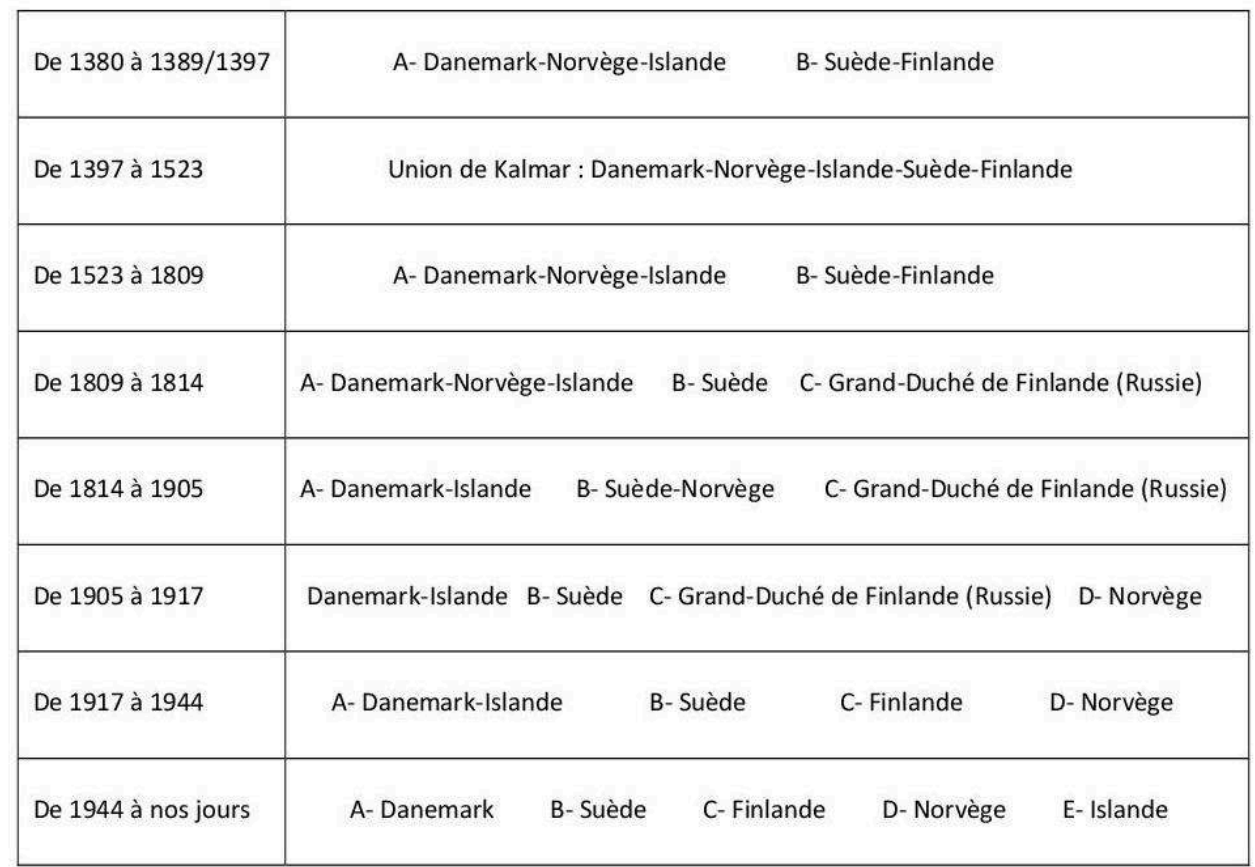

7 Ce régionalisme politique a été indéniablement renforcé par des avancées en matière d'intégration économique et démographique. Par exemple, 20 à $30 \%$ des échanges de chacun de ces pays concernent aujourd'hui les autres pays nordiques, ce qui témoigne d'une forte interpénétration par le voisinage, compte tenu de la faiblesse intrinsèque de chacune de ces économies et de leur propension à émettre sur le marché des produits similaires (produits forestiers par exemple). En effet, ces pays ne se classent dans l'échiquier mondial de l'OMC qu'entre la $32^{\text {ème }}$ place (pour la Suède) et la $42^{\text {ème }}$ place (pour la Finlande) des exportateurs de marchandises (Islande non comprise) et entre les $31^{\text {ème }}$ et $43^{\text {ème }}$ places des importateurs en 2017. Par ailleurs, de nombreux Nordiques résident aujourd'hui dans un pays où ils ne sont pas nés, les Finlandais notamment qui forment historiquement les plus gros bataillons des étrangers résidant en Suède. Aussi, les Nordiques sont longtemps restés les seuls étrangers dans les différents pays du Nord, avant l'arrivée massive lors des dernières décennies, de populations d'origine plus lointaine, venant d'Europe centrale, d'Asie ou d'Afrique de l'Est (Simon (éd.), 2015).

8 Pour autant, le régionalisme nordique n'est pas allé au-delà de simples relations de bon voisinage. Si le projet politique a capoté dès la fin du XIX ${ }^{\text {ème }}$ siècle, la tentative de constituer une union douanière après la Seconde Guerre mondiale n'a pas connu un meilleur sort (Rogé, 1956 ; Mousson-Lestang, 1990). Pourtant, dans le sillage d'unions douanières régionales comme le Benelux, dont le traité entre les trois États fondateurs avait été signé dès septembre 1944, une commission sous la présidence de C.V. Bramsnaes, directeur de la Banque Nationale du Danemark, avait été constituée pour jeter les bases d'une union douanière nordique. Elle publia un rapport très favorable en 
janvier 1950, mais rien de concret n'en sortit, en raison surtout des réserves émises par les pays les moins industrialisés, la Norvège en particulier qui estimait y voir un cheval de Troie propice à l'intrusion massive de produits suédois sur son marché national. Le projet Uniscan, élargi à la Grande-Bretagne, ne connut pas un meilleur sort. La relance d'une union douanière, sous le nom de Nordek, limitée cette fois aux seuls pays nordiques, fut un nouvel échec, l'Islande et surtout la Finlande s'abstenant de participer au projet en 1970. En fait, les espoirs d'une union strictement nordique ne résistaient pas à des ambitions d'intégration européenne et finalement, pour reprendre les termes d'un texte de L. Sanders (1996) «si leur coopération est ancienne à de nombreux égards, c'est au travers d'associations plus larges que les pays du Norden sont parvenus à renforcer leurs propres liens : l'AELE, puis l'EEE et tout récemment l'UE ", comme s'il avait fallu de plus vastes horizons et de plus fortes impulsions pour parvenir à une entente plus solide. Mais ces contraintes régionales eussent été sans conséquences si elles n'avaient pas été systématiquement contraires au projet régionaliste, nuisant au total à la consolidation du Norden, comme à son incorporation dans de plus vastes ensembles.

\section{Une régionalisation non conforme au projet régionaliste}

\section{La puissance des polarisations externes}

Rappelons tout d'abord que les économies nordiques disposent de marchés intérieurs limités et qu'elles ont trouvé dans les besoins du monde extérieur de puissants leviers de développement (Hodne, 1975 ; Wettergreen, 1978). La part des exportations dans les PNB est donc beaucoup plus élevée que dans la majeure partie des pays de même niveau de développement, ces exportations trouvant dans un voisinage proche, mais extérieur à l'espace nordique, l'essentiel de leurs clients. C'est ainsi que le marché britannique fut décisif pour les pays nordiques occidentaux (Danemark, Norvège) pendant plus d'un siècle. Par exemple, au début des années 1950, le tiers des exportations danoises (sous la forme essentiellement des produits de l'élevage) ou le cinquième des exportations norvégiennes étaient encore destinés au marché britannique. Le Royaume-Uni est resté le premier client de la Norvège sur toute la période 1866-1969, si on veut bien en exclure les années des conflits mondiaux. Au total, le Danemark a très largement profité de l'ouverture du marché britannique aux produits agricoles (suite à l'abandon des Corn Laws en 1846, facilitant l'importation des céréales, puis des produits de l'élevage danois); la Norvège a fini par imposer sa flotte marchande, avec l'ouverture du commerce britannique aux flottes étrangères (suite à la suppression de l'acte de navigation en 1849).

Cela dit, le marché britannique n'eut pas les mêmes effets pour tous les pays nordiques. La Finlande par exemple, tournée vers la Russie de 1809 à 1917, puis conduite par les accords postérieurs à la Seconde Guerre mondiale à écouler une bonne part de ses exportations vers l'URSS, est restée un pays balte, plus qu'un pays de l'Europe du nordouest. Par ailleurs, les centres d'impulsion extérieurs au monde nordique ont notoirement évolué au fil des ans, aux dépens du Royaume-Uni et à l'avantage de l'Europe communautaire et singulièrement de l'Allemagne. D'où les fracturations $d u$ bloc nordique et surtout les hésitations en matière d'intégration régionale, alors même 
que l'effort de structuration du Norden restait fragile et inachevé. En revanche, les espoirs placés dans le Norden rendaient difficile une intégration complète à l'œuvre communautaire.

11 On comprend donc pourquoi les pays nordiques ont d'abord penché pour le choix britannique de l'association européenne de libre-échange, effective suite à la signature de la convention de Stockholm du 4 janvier 1960. Le Danemark, la Norvège, la Suède se rangèrent parmi les membres fondateurs, vite rejoints par l'Islande en 1970. On devine aussi pourquoi la Finlande est restée en retrait: de simple associée en 1961, elle ne devint membre à part entière qu'en 1986.

Puis le basculement des centres de gravité, entériné par l'entrée en Communauté du Royaume-Uni à compter du $1^{\mathrm{er}}$ janvier 1973 a changé considérablement la donne. C'est le début du grand «schisme scandinave» (Mousson-Lestang, 1990), puisque le positionnement des États nordiques allait durablement diverger entre les États « atlantiques » et les États baltes, ces derniers, Suède en tête, voyant dans la CEE la «branche économique de l'OTAN », pour reprendre une formule du Premier ministre suédois, Olof Palme. Double schisme en vérité, puisque le Danemark, suite à un référendum favorable, intègre la CEE en même temps que le Royaume-Uni, alors que la Norvège en est empêchée, suite à un référendum défavorable. Il faut voir dans la formule un peu abrupte d'Olof Palme, le souci de préserver la neutralité suédoise, à mettre en balance avec la neutralité finlandaise, les deux s'appuyant l'une sur l'autre pour maintenir un espace de paix dans le Nord européen. C'est cette préoccupation qui avait empêché la Suède d'adhérer au traité de l'Atlantique Nord en 1949, alors que le Danemark, la Norvège et l'Islande n'avaient pas eu ces scrupules, faisant incidemment capoter tout espoir de défense mutuelle des pays du Nord.

\section{Intégration européenne et impossible cohésion nordique}

13 L'élargissement de l'Union européenne au $1^{\text {er }}$ janvier 1995 pouvait apparemment redonner de la cohérence à cette Europe nordique. En fait, l'intégration de la Suède et de la Finlande, préparée par des accords d'association depuis 1972-1973, s'est faite dans un climat de profonde ambiguïté. Pour la Finlande, il s'est agi par cette adhésion à l'Europe de recouvrer pleinement sa liberté, en se libérant enfin de la tutelle russe, voire de celle plus ancienne de la Suède, par un acte fondateur de sa propre liberté de mouvement (Simoulin, 2000). D'où le succès indiscutable du référendum préalable à son adhésion, à plus des deux tiers des suffrages exprimés. Pour la Suède au contraire, il s'est agi d'un choix imposé par la nécessité, à un moment crucial de son histoire, c'està-dire à la fin d'un cycle favorable à la social-démocratie et au fameux "modèle suédois ». L'adhésion à l'Union fut d'ailleurs présentée comme un moyen de régler tous les problèmes internes et c'est dans ce contexte que les Suédois se prononcèrent à une courte majorité (à 52,3 \% des voix) pour leur entrée dans l'Union. Par ailleurs, beaucoup d'eurosceptiques mettaient en doute la capacité de l'Union à préserver un haut niveau de développement pour leur propre pays, alors même que les standards économiques de la plupart des pays membres étaient largement inférieurs aux leurs. L'Union dut d'ailleurs consentir quelques aménagements de sa politique de cohésion régionale, en inventant un nouvel objectif (le 6 pour la période 1994-1999), destiné aux régions périphériques au PIB élevé mais handicapées par des difficultés spécifiques, en particulier le très faible peuplement. C'était précisément le cas du Nord suédois et finlandais. Cela dit, les réticences à l'égard d'une intégration politique plus poussée ont 
toujours subsisté en Suède, comme le signale le référendum de 2003 sur le passage à l'euro, assez nettement défavorable (à 55,9 \% des voix).

Quant à l'Europe du Nord "atlantique ", elle n'a pas donné les preuves d'une meilleure cohésion. La majorité des Danois est restée dans la logique du marché commun, sans réelle contrepartie pour aller plus avant dans l'intégration. Leur refus du traité de Maastricht en 1992 (à 50,7 \% des voix) a mis en lumière cet esprit de résistance, dont les Européens durent s'accommoder par les accords d'Edimbourg qui accordaient aux Danois quatre options de retrait à leur participation à l'union économique et monétaire. C'est à ce prix qu'un nouveau référendum l'année suivante a fini par faire accepter le traité de Maastricht, non sans quelques réserves, puisque les Danois firent jouer l'une de leur option de retrait en refusant leur entrée dans la zone euro par un nouveau référendum en 2000 (53,2 \% des voix). Ils sont même allés jusqu'à refuser de suspendre leur option de retrait à la coopération judiciaire et policière avec les autres États de l'Union en 2015 (à la hauteur de 53,1 \% des voix). Il est vrai que le Danemark a des difficultés pour se positionner face à l'Union, dans la mesure où ses terres d'« outre-mer » sont plutôt hostiles à l'idée européenne. Les Féroé n'ont jamais fait partie de l'Union et le Groenland a estimé finalement que son appartenance à l'Europe devait être remise en cause, suite à une consultation référendaire en 1982, le Groenland sortant finalement de la communauté européenne en 1985. Mais c'est la Norvège qui provoqua au bout du compte la fracturation la plus nette de cette Europe du Nord "atlantique». Engagée dans des négociations qui devaient déboucher sur son intégration en même temps que celles de la Suède et de la Finlande, elle s'en est trouvée empêchée par un référendum à nouveau défavorable en novembre 1994. Il est certain que les politiques intégrées de l'Union, en matière agricole et halieutique, ont pesé très lourd dans cette décision, le pays se contentant finalement de l'Espace économique européen, au même titre que l'Islande, un moment tentée de négocier son adhésion à l'Union, puis la suspendant à peu près pour les mêmes raisons (tableau 2).

Tableau 2. Les États Nordiques et l'intégration européenne : un positionnement en ordre dispersé.

\begin{tabular}{|c|c|c|c|c|}
\hline & AELE & \begin{tabular}{|l|l} 
EEE & Espace Schengen
\end{tabular} & Union européenne & Zone Euro \\
\hline \multicolumn{5}{|l|}{ Danemark } \\
\hline \multicolumn{5}{|l|}{ Finlande } \\
\hline \multicolumn{5}{|l|}{ Islande } \\
\hline Norvège & & & & \\
\hline Suède & & & & \\
\hline
\end{tabular}

\section{Les hésitations d'un régionalisme d'inspiration européenne}

Il est donc difficile de prendre en compte le Norden, dans son acception traditionnelle, dans les politiques régionales de l'Union. Il est de toute façon bien trop riche pour bénéficier des politiques de convergence qui sont actuellement réservées dans le Nord massivement aux États baltes. Quant aux politiques de coopération, structurées par le programme Interreg, dont c'est la cinquième version entre 2014 et 2020, elles concernent les États du Norden pour 7 programmes de coopération transfrontalière, mais à l'échelle de régions infraétatiques (volet Interreg V-A). Plus ambitieux dans ses objectifs, serait le volet Interreg B qui concerne tout ou partie des États en les associant autour d'espaces transnationaux de grande envergure. Le Norden est concerné par trois 
des quinze espaces transnationaux Interreg V-B qui ont été définis pour la période 2014-2020 : la mer du Nord, la périphérie nordique et arctique, la mer Baltique. Cette affectation suggère des tropismes disparates, entre le bassin maritime de l'Europe du nord-ouest, les marges arctiques et leurs enjeux face au réchauffement climatique et l'accès aux ressources qui leur est associé, et la Baltique que l'Union européenne souhaiterait promouvoir au titre de véritable mer intérieure de l'Europe du Nord, une sorte de centre de gravité de ses projets régionalistes. Mais l'espace baltique, aux yeux des Scandinaves, a souvent été considéré comme une marge, une bordure, la perte de la Finlande par la Suède en 1809 et surtout la période de glaciation soviétique après la Seconde Guerre mondiale renforçant encore cette impression. Du reste, les Scandinaves s'obstinent à parler de l'Østersjøen (la mer de l'Est) pour évoquer la Baltique, les États baltes étant à leurs yeux les Østerstatene. Il subsiste donc un sensible décalage entre les visions du Norden et les visions bruxelloises qui tentent de fédérer autour d'elles tout ou partie des États riverains, visions qu'on retrouve dans la création en 2009 de la macrorégion de la mer Baltique, concernant cette fois la totalité des États riverains, à l'exception notable de la Russie. Ces initiatives rencontrent l'enthousiasme de quelques intellectuels (tel $\mathrm{T}$. Christiansen, considérant que la région baltique « pourrait servir de livre de recettes pour d'autres espaces européens ou mondiaux, où l'action politique dirigée vers l'échelle étatique ou nationale a fait son temps » - cité dans Escach, 2011) ou, à l'échelle locale, des réseaux de villes promptes à ressusciter les comptoirs hanséatiques (Escach, 2012). Il faut pourtant admettre que c'est oublier un peu vite la permanence des tensions et des conflits à propos de cette mer où se combinent la densité des usages et la pesanteur de considérations géopolitiques de dimension extrarégionale (Escach, 2016; Bayou, 2018). C'est surtout oublier le Norden et la complexité de son contexte géographique, élargi à ses dimensions atlantique et arctique. Par ailleurs, les États nordiques riverains de la Baltique n'ont pas attendu la construction européenne pour coordonner leurs efforts sur des objectifs limités à la protection environnementale de la mer Baltique, avec la convention d'Helsinki de 1974 qui a réussi à intégrer l'URSS au titre des parties contractantes. Cette convention a été réactualisée en 1992, avec dix parties contractantes, dont la Russie et l'Union européenne. On devine ici une stratégie d' « entre-deux ", évitant l'affrontement entre des blocs supposés hostiles à propos d'objectifs précis et consensuels. Cette stratégie des États nordiques qui les rend indispensables au titre de passerelles entre deux mondes a été répétée par le Conseil nordique, lorsqu'il a mis en place un conseil des États de la mer Baltique dès 1992, impliquant la Russie et les États nordiques (Vauguet, 2008). Il s'est agi en quelque sorte d'éviter la marginalisation des questions nordiques, tout en intégrant la Russie pour en régionaliser les préoccupations. On retrouve la même stratégie avec le conseil euro-arctique de la mer de Barents. L'Union européenne étant représentée dans ces deux conseils, la stratégie nordique consiste donc à pousser l'Europe à se préoccuper des intérêts nordiques dans leur cadre régional effectif, tout en obligeant l'« empire continental russe » à considérer le point de vue de ses voisins lorsqu'il est en recherche de ses nécessaires débouchés maritimes. 


\section{Quelques facteurs géographiques pouvant expliquer cette discordance entre régionalisme et régionalisation}

\section{Le poids de l'échelle locale}

16 Peut-on alors mobiliser des facteurs géographiques plus infrastructurels pour expliquer cette discordance entre régionalisme et régionalisation et finalement cette (dé)union, à la fois externe et interne, des pays nordiques (Meyer, 2018) ? Comme rappelé dès l'introduction de la présente étude, on avance ici dans un univers incertain, fait de suppositions plus que de certitudes, les idées avancées restant encore à l'état d'hypothèses. Signe qui ne trompe guère : la littérature géographique est plus encline à étudier la Scandinavie ou le Norden de manière globale et intégrée chez les auteurs étrangers, comme s'il fallait une mise en surplomb ou une mise à distance pour dégager les lignes de force des spécificités scandinave ou nordique (John, 1984), alors que les ouvrages sous la responsabilité totale ou partielle d'auteurs "locaux" sont plus circonspects, accordant toute leur place aux États, au-delà de quelques incontournables généralités (Varjo, Tietze, 1987). En conclusion du dernier ouvrage cité, l'« ensemble des ensembles » qui propose un découpage régional du Norden, ne s'affranchit guère des frontières nationales, considérant implicitement que la structure territoriale de chacun des États prime sur toute autre forme d'organisation. Mais faisant partie du lot des observateurs étrangers, proposons tout de même quelques généralisations et sans doute approximations à propos de la question que nous nous posons.

Tout d'abord, l'échelle locale nous semble primordiale dans ce monde pulvérisé et relativement isolé. Le bygd, terme difficile à traduire mais qui peut être assimilé en première approche à la commune de tradition rurale, est l'élément fondateur de la construction territoriale. Du reste, les plus petits noyaux agglomérés de peuplement, dont les seuils les plus modestes peuvent descendre jusqu'à 200 habitants, rejoignent sans peine ce cadre dans lequel s'est longtemps épanouie une société rurale et agraire. C'est là que le patrimoine "authentique » du monde nordique s'est constitué, c'est aussi là qu'il est souvent magnifié par sa mise en valeur muséographique, dans ces folkemuseum de plein air, si nombreux en Scandinavie. Or, pour les Nordiques, le meilleur cadre de protection de cette échelle locale reste l'État dont la taille semble évidemment décalée par rapport à l'échelle locale, mais dont il ne faut jamais oublier la dilatation territoriale sur des espaces de faible usage, alors même que sa population reste de taille modeste, à l'échelle d'un État « régional ». Protecteur des particularismes locaux, soucieux d'équilibre en matière de politique régionale, l'État est donc tout naturellement requis pour la protection des cellules de base de la société nordique. Les transferts de souveraineté qu'exige l'Europe intégrée sont donc ressentis comme une atteinte au bon fonctionnement de l'échelle locale : amoindrir l'État revient à affaiblir la cellule du bygd. Ce ressenti se vérifie en de maints exemples, comme en Norvège, lorsqu'une corrélation assez étroite apparait entre les espaces où domine la langue " patrimoniale » du landsmål (ou nynorsk), c'est-à-dire une langue enracinée dans son identité rurale et débarrassée d'influences étrangères, et les espaces hostiles à l'intégration européenne, notamment dans l'Ouest, alors que l'autre langue officielle, le riksmål ou bokmål, proche lexicalement du danois, correspond aux espaces métropolitains favorables à l'Europe (sauf dans le Nord où les déterminants 
périphériques et économiques dominent pour expliquer le refus de l'intégration). La stabilité des positionnements territoriaux est assez remarquable lors des deux référendums norvégiens de 1972 et de 1994 qui ont posé la question fondamentale de l'intégration européenne avec une très forte mobilisation de la population $(79,2 \%$ des inscrits en 1972 et $89 \%$ en 1994). Avec un léger effritement du front du refus (52,2\% des voix en 1994 , contre $53,5 \%$ en 1972), une seule province, dans le sud-est, a changé d'avis, en choisissant le « oui ». Oslo a maintenu sa confiance dans l'Europe (66,6 \% de " oui ", contre $66,5 \%$ en 1972), à l'instar de trois autres provinces, toutes dans le sudest. Dans l'Ouest, le refus est resté net $(68,2 \%$ au pays des fjords, dans le Sogn og Fjordane), tout comme dans le Nord (avec plus de $70 \%$ de «non» dans les trois provinces du Nord-Norge, soit un pourcentage à peu près identique à celui de 1972).

N'oublions pas que les États nordiques sont aussi des États unitaires, expression de nations bien affirmées (sauf pour les Sames). Leur modestie les exonère de toute idée de puissance, à laquelle ils substituent sur le plan international une diplomatie faite de neutralité (pour la Suède et la Finlande qui maintiennent leur refus d'adhérer à l'otan, malgré des accords de partenariat signés en 2014) et d'engagement à l'égard des pays les plus démunis, engagement par lequel ils cultivent avec patience et persévérance une sorte de «soft power » à l'échelle mondiale. D'où la méfiance pour la formation de grands ensembles dans lesquels ils ne pourraient guère peser. Pour reprendre une expression de l'écrivain norvégien B. Bjørnson (cité dans Kersaudy, 1980), «il n'est jamais rien venu de bon que des petites nations - ou des grandes...quand elles étaient petites ». Enfin, les États nordiques sont longtemps apparus comme les garants du bienêtre social et des avancées sociétales. Or le modèle est en crise depuis les années 1980 et cette crise est souvent confondue avec les conséquences de l'intégration à une Europe jugée trop libérale. On impute donc à l'Europe une partie des causes des difficultés du modèle, alors que des raisons endogènes peuvent être convoquées: évolution des structures démographiques, montée du salariat tertiaire et déspécialisation des tâches productives subalternes, internationalisation des entreprises, essoufflement de la croissance des besoins de consommation (Durand (éd.), 1994). C'est une des clés d'explication de l'hostilité à l'Europe de l'aile la plus à gauche de la société, de la fracturation profonde de la social-démocratie à son égard et des réserves des mouvements écologistes à propos d'une Europe jugée trop éloignée des préoccupations environnementales.

\section{Europe et ouverture au monde}

Pourtant, les Nordiques soient loin d'être refermés sur eux-mêmes, puisqu'ils ont baigné depuis ces 150 dernières années dans une large économie d'ouverture. Toutefois, cette économie d'ouverture fut d'abord atlantique, autant pour ses partenaires de l'échange que pour les terrains d'accueil de ses populations migrantes (l'Europe nordique a vu partir 2,6 millions de personnes entre 1860 et 1914, principalement en Amérique du Nord). Il en a résulté de solides relations de part et d'autre de l'Atlantique Nord dont l'une des meilleures illustrations aujourd'hui est la remarquable maitrise de l'anglais, sorte de lingua franca de la plupart des Nordiques. Même si cette maîtrise est largement soutenue par un apprentissage précoce en milieu scolaire, elle est en phase avec une histoire et une culture largement atlantiques. En revanche, la mondialisation contemporaine change quelque peu la donne, non seulement en termes d'équilibres des comptes à l'égard du monde extérieur, mais aussi 
en termes d'accueil de nouvelles populations, dont les origines en quelques décennies, ont été profondément renouvelées. Au Danemark, les immigrés non-occidentaux et leurs descendants ne formaient que $1,5 \%$ de la population en 1985 , ils étaient $7 \%$ en 2013. En Norvège, plus de 552000 personnes en 2010 étaient à ranger dans la catégorie des immigrés, dont près de 200000 étaient nées sur le continent asiatique. En Suède, la population immigrée est passée de 6,6\% de la population en 1970 à $14 \%$ en $2011(1,3$ million de personnes sur une population totale de 9,3 millions d'habitants). De plus, la concentration métropolitaine de cette population renforce sa visibilité aux yeux des «autochtones ». En Norvège, les deux provinces d'Oslo-Akershus, soit le quart de la population nationale, concentrent $42 \%$ de la population immigrée (et même la moitié des immigrés asiatiques). Au total, la région métropolitaine s'avère très cosmopolite, avec un habitant sur cinq d'origine immigrée (et même plus d'un habitant sur quatre pour la seule ville d'Oslo). De même en Suède, les trois comtés concernés par les agglomérations de Stockholm, Göteborg et Malmö concentrent à eux seuls près des deux tiers des immigrés établis sur le territoire suédois (Simon (éd.), 2015). Dès lors, la question migratoire s'impose dans le positionnement des différents partis politiques. En Norvège, le fremskrittsparti (Parti du Progrès) a fait un bond spectaculaire au point de frôler le quart des votes exprimés aux élections législatives de 2009 et finalement de participer au gouvernement en 2013. Résolument libéral dans ses idées économiques, volontiers critique à l'égard des impôts, il est aussi hostile à la nouvelle immigration et c'est sur ce dernier volet qu'il a assuré son succès. Critique à l'égard de l'Europe, il oblige la droite traditionnelle à mettre un sérieux bémol à ses convictions européennes. Le cas norvégien n'est pas isolé, puisqu'on retrouve les mêmes courants de pensée au Danemark (avec le Parti Populaire Danois), en Suède (avec le Parti des Démocrates de Suède) ou en Finlande (avec le Parti des Vrais Finlandais, c'est-à-dire des Finlandais de "base », inspirés par des valeurs rurales et agrariennes). On retrouve ici l'importance de l'échelle locale, où se maintiennent les valeurs nordiques, à l'inverse des villes, jugées trop ouvertes sur le monde.

20 En même temps, ces villes semblent bien modestes à l'échelle du monde contemporain. En passant en revue les trois critères de la nodalité, de la centralité et de la polarité, les villes nordiques trahissent en effet des signes incontestables de fragilité. La nodalité qui est affaire de synapses et de carrefours, donc de réseaux au service de flux matériels, est bien révélée par le poids des villes-ports. Or, on sait depuis longtemps que Hambourg joue un rôle majeur dans l'ouverture au monde de la Baltique et de la Scandinavie (Weigend, 1956 ; Ouren, 1976). Ce rôle hérité des traditions hanséates, s'est très largement renforcé avec la mise en place des réseaux de lignes conteneurisées, les ports nordiques devant céder les uns après les autres leurs escales de lignes directes pour les remplacer par des services de feedering au départ de la Northern Range et singulièrement de Hambourg (Escach, Serry, 2013). Ainsi, au fur et à mesure que les économies se mondialisent, les transports maritimes se régionalisent (Serry, 2018), pour les frets les plus nobles, à savoir les marchandises diverses conteneurisées. C'est un signe, sinon de vulnérabilité, du moins de dépendance, auquel n'échappe qu'avec difficulté le seul port de Göteborg (Guillaume, 2012).

21 Quant à la centralité et à la polarité, elles sont affaire d'économies d'agglomération, c'est-à-dire d'avantages économiques, directs ou indirects, tirés du regroupement des populations et de leurs activités. Il convient ici de distinguer les effets de taille, permettant d'économiser sur les coûts unitaires de production et facilitant l'éveil et 
l'équilibre des services nécessitant un volume suffisant de clientèle, et les effets de coexistence, provoquant par réactions en chaîne, la diversification et l'attractivité des activités. Les premiers sont à ranger dans le registre de la centralité, les seconds dans celui de la polarité. Or la centralité reste contrainte dans le monde nordique, d'une part en raison des spécificités de sa révolution industrielle, les effets de taille ayant été contrariés par la grande dispersion des facteurs matériels de la production (eau, bois, ressources minérales), provoquant ainsi la multiplication des petits centres industriels spécialisés (Hansen, 1980), d'autre part par le faible contingent des populations à urbaniser. Quand bien même le regroupement des populations urbaines est élevé dans les régions centrales (jusqu'à $30 \%$ de la population danoise pour la région de l' Hovedstaden, voire plus de $60 \%$ pour celle de Reykjavik en Islande), le poids des villes primatiales est limité à des agglomérations de 1 à 1,5 million d'habitants. Quant à la polarité, elle s'exprime au travers des services aux entreprises les plus élaborés et aux réseaux hiérarchisés à l'intérieur de ces mêmes entreprises, dont la mise en place est facilitée par la communication instantanée de l'information. Cette mise en réseaux repose sur des villes en archipel, dont les têtes (les villes globales) sont les seules à détenir les plus hauts niveaux de pouvoir (les sièges sociaux et les services qui leur sont associés). Si l'on suit les classements du GaWC (Globalization and World Cities Research Network), qui reprend cette thématique réticulaire pour une centaine d'entreprises dans le secteur de la finance, de l'expertise, de l'assurance, de la publicité ou de l'immobilier, on obtient un classement des plus grandes villes mondiales en villes Alpha, Beta, Gamma, High Sufficiency et Sufficiency. On constate qu'il n'y a qu'une seule ville Alpha dans les États nordiques, Stockholm, en queue de peloton ( $38^{\text {ème }}$ en 2016 sur 49 dans le monde) et en perte de vitesse relative (27 $7^{\text {ème }}$ en 2000). Les autres villes métropolitaines ne sont que des villes Beta: Copenhague au $53^{\text {ème }}$ rang, Helsinki au $59^{\text {ème }}$, Oslo au $65^{\text {ème }}$. Au total, ce sentiment de faiblesse et de mise en périphérie, ne facilite pas l'adhésion à de plus vastes ensembles, puisqu'elle pourrait faire craindre une rapide satellisation.

\section{Conclusion}

Le Norden, terme finalement assez méconnu hors de l'Europe du Nord, doit composer avec d'autres qualificatifs, la Scandinavie bien sûr, mais aussi la Fennoscandie ou plus récemment, la Baltoscandie, comme si la terminologie dépendait du contenu ou pire encore, des intentions qu'on voulait y mettre. Le dessin des limites dépend donc très étroitement du dessein des projets. Cette instabilité des mots révèle bien évidemment la complexité de la chose, la multiplicité de ses éclairages et la diversité de ses pôles d'attraction (Neumann, 1994). On est donc loin des derniers stades aboutis de la construction régionale, tels qu'envisagés par Hettne et Söderbaum (2000), conduisant à une entité distincte de son environnement extérieur et doué d'un certain niveau de légitimité, reconnu à la fois par la société régionale (si elle existe) et par le monde qui l'entoure. Dans le tome de la Géographie universelle consacré assez curieusement à l'attelage entre l'Europe du Nord et l'Europe médiane, J.-P. Marchand (1996) suggère à propos de l'Europe du Nord, une explication faite d'un sentiment d'isolement, d'autant mieux ressenti qu'il est multiscalaire : «on passe ainsi de l'échelle subcontinentale à celle des rochers et des promontoires, par une descente en fractale entre les différents niveaux de cassure qui participent au morcellement insulaire de l'Europe du Nord et des liens que les populations doivent entretenir pour les surmonter ». Double tension 
donc entre l'isolement et la recherche du contact, qui est constitutive pour les Nordiques de leur appartenance au monde. Que cette tension cesse, par repli sur soi ou par adhésion à l'Autre et les Nordiques perdraient leur identité, par la marginalité dans le premier cas et la banalité dans le second.

\section{BIBLIOGRAPHIE}

BOYER R. (1992), « L'âge romantique (1800-1864) », in BATTAIL J.-F., BOYER R. \& FOURNIER V., Les sociétés scandinaves de la Réforme à nos jours, Paris, PUF, 596 p.

BAYOU C. (2018), « Tensions sécuritaires dans la région Baltique. Que reste-t-il de l'équilibre nordique? », Questions Internationales, 90, Paris, pp. 106-112.

CABOURET M. (1989), « Les fondements géographiques de la mise en valeur de la calotte polaire boréale en Fenno-scandie », Norois, 142, Poitiers, pp. 171-181.

CABOURET M. (1992), « Vers un recentrage de la région de l'Öresund », Norois, 156, Poitiers, pp. 369-388.

CHRISTIANSEN T. (1997), “A European Meso-Region? European Perspectives on the Baltic Sea Region", in New-Nationalism or Regionality, the restructuring of politicial space around the Baltic Rim, Stockholm, NordRefo, pp. 254-292.

DURAND J.-P. (éd.) (1994), La fin du modèle suédois, Paris, Syros, 285 p.

ESCACH N. (2011), « Doit-on renoncer à définir une "région baltique ?” ", Nouvelle-Europe, http : // www.nouvelle-europe.eu, $7 \mathrm{p}$.

ESCACH N. (2012), « Les réseaux de villes dans l'espace baltique : le mythe, "visée mobilisatrice" ou "identité de l'être"? », Nouvelle-Europe, http : //www.nouvelle-europe.eu, 9 p.

ESCACH N., SERRY A. (2013), « Les ports de la Baltique entre mondialisation des échanges et régionalisation réticulaire », Géoconfluences, Lyon, http://geoconfluences.ens-lyon.fr, 36 p.

ESCACH N. (2016), « Partage de la mer et nouveaux conflits géopolitiques en Baltique », Hérodote, 163, Paris, pp. 175-191.

FOL J.-J. (1978), Les pays nordiques aux XIX ème et XX ${ }^{\text {ème }}$ siècles, Paris, Nouvelle Clio, 327 p. GaWC (2016), Globalization and World Cities Research Network, Loughborough University, www.lboro.ac.uk/gawc.

GUILLAUME J. (2012), « Réorganisation maritimo-portuaire et développement d'une périphérie intégrée : l'exemple de l'Europe nordique », Norois, 223, Rennes, pp. 105-121.

HANSEN J.Ch. (1980), “Industristedenes plass i Norges bosettingsmønster”, Ad Novas, 17, Universitetsforlaget, $176 \mathrm{p}$.

HETTNE B., SÖDERBAUM F. (2000), “Theorizing the Rise of Regionness”, New Political Economy, 5, 3, pp. 457-474.

HODNE F. (1975), An Economic History of Norway 1815-1970, Oslo, Tapir, 549 p. 
JOHN B.S. (1984), Scandinavia, a new geography, London, Longman, 365 p.

KERSAUDY F. (1980), « Les relations internordiques contemporaines », Cahiers du Ciren, 1, Paris, pp. 17-29.

MARCHAND J.-P., RIQUET P. (dir.) (1996), Europe du Nord, Europe médiane, Paris-Montpellier, BelinReclus, $480 \mathrm{p}$.

MAREÏ N., RICHARD Y. (dir.) (2018), Dictionnaire de la régionalisation du monde, Paris, Atlande, 351 p.

MEYER T. (2018), « Les pays nordiques : (dés)union septentrionale », Carto, 49, pp. 26-28.

MOUSSON-LESTANG J.-P. (1990), La Scandinavie et l'Europe de 1945 à nos jours, Paris, PUF, 206 p.

NAUWELAERS C., MAGUIRE K. \& MARSAN G.A. (2013), “The Case of Öresund (Denmark-Sweden), Regions and Innovation: Collaborating across Borders", OECD Regional Development Working Papers, $21,59 \mathrm{p}$.

NEUMANN I. (1994), “A Region-Building Approach to Northern Europe”, Review of International Studies, 20, 1, pp. 53-74.

OUREN T. (1976), "The Role of the Large European Ports in the Shipments of Cargo between Norway and Transoceanic Countries", Norsk Geografisk Tidsskrift, Oslo, pp. 171-178.

PARENT J. (1970), Le modèle suédois, Paris, Calmann-Lévy, 310 p.

ROGÉ F. (1956), « Le marché commun scandinave et ses perspectives », Etudes et Conjoncture, Paris, pp. 1080-1095.

SANDERS L. (1996), « La modernité du Norden », in MARCHAND J.-P., RIQUET P. (dir.) (1996), Europe du Nord, Europe médiane, GU, Belin-Reclus, pp. 152-165.

SERRY A. (2018), Le transport maritime en mer Baltique, note stratégique et prospective, Le Havre, Devport-Sefacil-Idées, $48 \mathrm{p}$.

SIMON G. (éd.) (2015), Dictionnaire des migrations internationales, approche géohistorique, Paris, A. Colin, $808 \mathrm{p}$.

SIMOULIN V. (2000), «L'Union européenne au regard des pays nordiques », Paris, Les Etudes du CERI, Sciences Po, 66, $33 \mathrm{p}$.

SIMOULIN V. (2003), « Le Conseil nordique : vecteur identitaire ou bureaucratie perdue? », Nordiques, 1, Caen, pp. 11-32.

VARJO U., TIETZE W. (eds.) (1987), Norden, Man and Environment, Gebruder Borntraeger, Berlin, Stuttgart, $535 \mathrm{p}$.

VAUGUET F. (2008), « La Baltique, exemple de coopération régionale? », Nouvelle-Europe, http : // www.nouvelle-europe.eu, $7 \mathrm{p}$.

WEIGEND G.G. (1956), “The Problem of Hinterland and Foreland as illustrated by the Port of Hamburg", Economic Geography, 32, pp. 1-16.

WETTERGREEN Kj. (1978), “Konjunkturbølger fra utlandet i norsk økonomi”, Oslo, Samfunnsøkonomisk Studier, 36, Oslo, SSB, 141 p.

ZORGBIBE Ch. (1968), Les États-Unis scandinaves, Paris, Pedone, $144 \mathrm{p}$. 


\section{RÉSUMÉS}

Après avoir défini les termes qui sont à la base de la réflexion de cet article, l'auteur insiste sur les fondements de l'unité du Norden qui dépendent en grande partie de considérations culturelles et politiques à ranger dans les catégories du régionalisme. Ce régionalisme se heurte néanmoins à de fortes polarisations externes qui peuvent se comprendre selon des processus économiques, actuellement repris ou couverts par l'intégration européenne. Les résistances à cette intégration sont explorées en troisième partie sous le regard de quelques facteurs géographiques.

After defining the terms on which this paper is based, the author describes the foundations of Norden's unity that depend on cultural and political categories to be classified in regionalism. Nevertheless, he suggests also their limitations, which are partly related to strong external and economic polarizations, linked to regionalization process and revealed by the European integration. Geographical factors of explanation are mentioned in the third part of this paper.

\section{INDEX}

Mots-clés : régionalisme, régionalisation, intégration européenne, Norden, Scandinavie, Europe nordique

Keywords : regionalism, regionalization, European integration, Norden, Scandinavia, Northern Europe

\section{AUTEUR}

\section{JACQUES GUILLAUME}

Professeur émérite, Igarun, Université de Nantes, jacques.guillaume@univ-nantes.fr 